\title{
La conciliación entre libertad y necesidad en la Ética de Spinoza*
}

[Artículos]

Alejandro Villamor Iglesias ${ }^{* *}$

Recibido: $26 / 10 / 2020$

Aprobado: 16/12/2020

Citar como:

Villamor, A. (2021). La conciliación entre libertad y necesidad en la Ética de Spinoza. Análisis, 53(98). https://doi.org/10.15332/21459169.6236

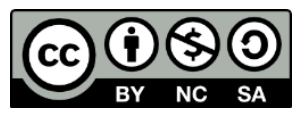

\section{Resumen}

Spinoza presenta en su Ética una ontología en virtud de la cual el hombre no es más que un modo determinado por otros modos. Nos encontramos, así pues, con una concepción de la naturaleza del hombre que ha sido considerada tradicionalmente como determinista. Tal y como denunciaron algunos autores como Jacobi o Tschirnhaus, de esta concepción según la cual toda acción humana es necesaria y está determinada de antemano, se seguiría la ausencia de la responsabilidad moral. Ahora bien, lejos de abandonarnos en un mundo amoral, en la propuesta de Spinoza no sólo está presente una determinada concepción de la libertad, sino también una concepción normativa de la ética. En consecuencia, en el presente trabajo se mostrará la senda seguida por el

\footnotetext{
* Artículo de reflexión.

** Máster en Formación de Profesorado de la Universidad de Santiago de Compostela, España. Máster en Lógica y Filosofía de la Ciencia de la Universidad de Salamanca, España. Actualmente ejerce como profesor de Filosofía de Educación Secundaria en la Comunidad de Madrid (España). Correo electrónico:

alejandrovillamoriglesias@yahoo.es; ORCID: https://orcid.org/0000-0001-7265-7528
} 
autor de la Ética para llegar a la conclusión de que libertad y necesidad son conceptos compatibles. Esto será posible tras abordar consecutivamente la concepción antropológica, ontológica y ética spinoziana.

Palabras clave: Ética, libertad, "libertad necesaria”, necesidad, Spinoza.

\section{Reconciling freedom and necessity in Spinoza's Ethics}

\section{Abstract}

Spinoza presents in his Ethics an ontology by virtue of which man is nothing more than a mode determined by others. Therefore, we find ourselves with a conception of man's nature that has traditionally been considered deterministic. As some authors have denounced, such as Jacobi or Tschirnhaus, this conception, according to which all human action is necessary and is determined in advance, the absence of moral responsibility would follow. However, far from abandoning us to an amoral world, in Spinoza's proposal there is present not only a certain conception of freedom, but also a normative conception of ethics. Consequently, this paper will show the path followed by the author of the Ethics to reach the conclusion that freedom and necessity are compatible concepts. This will be possible after addressing consecutively the anthropology, ontology and ethics conception of Spinoza.

Keywords: Ethics, freedom, "necessary freedom”, necessity, Spinoza.

\section{A conciliação entre liberdade e necessidade na Ética de Spinoza}

\section{Resumo}

Spinoza apresenta em sua Ética uma ontologia em virtude da qual o homem é apenas um modo determinado por outros modos. Por isso, nós 
nos deparamos com uma concepção da natureza do homem que tem sido tradicionalmente considerada determinista. Conforme denunciado por alguns autores como Jacobi ou Tschirnhaus, dessa concepção segundo a qual toda ação humana é necessária e determinada de antemão, seguiria a ausência de responsabilidade moral. Agora, longe de nos abandonar num mundo amoral, a proposta de Spinoza não só apresenta uma certa concepção de liberdade, mas também uma concepção normativa de ética. Consequentemente, este artigo mostrará o caminho seguido pelo autor da Ética para chegar à conclusão de que liberdade e necessidade são conceitos compatíveis. Isso será possível depois de abordar consecutivamente a concepção antropológica, ontológica e ética de Spinoziana.

Palavras-chave: Ética, liberdade, "liberdade necessária", necessidade, Spinoza.

\section{Introducción}

En sus Cartas a Mendelssohn, Jacobi advierte a Lessing del grave peligro al que nos avoca la filosofía racionalista (Barreiro, 1999, pp. 175-189). Abanderada por Spinoza, considerado el más consecuente de todos los filósofos racionalistas, esta filosofía nos conduce inevitablemente a un determinismo fatalista. En las Cartas, al igual que Jacobi, Lessing reconoce en la figura de Spinoza el autor que ha llevado a la filosofía a sus últimas consecuencias. La distancia entre ambos se impondrá a la hora de valorarlas. Mientras que Lessing no consigue otear el problema - pues afirma que no aspira "a que la voluntad sea libre"-, Jacobi reitera vehementemente que el determinismo de Spinoza conduce a un fatalismo nada deseable. Si solamente hubiera causas eficientes, nunca finales, según Jacobi los individuos simplemente seríamos meros "espectadores" de la naturaleza. La preocupación de Jacobi fue compartida, asimismo, por Tschirnhaus (Chaui, 2011, p. 269), quien afirma en sus cartas a Spinoza 
que, si consideramos la libertad como ausencia de determinación ${ }^{1}$, estamos abocados al fatalismo tan ironizado por Diderot en Jacques el fatalista. En definitiva, no fueron pocos los autores que consideraron que la propuesta ontológica pergeñada por Spinoza nos conduce inevitablemente a un pernicioso fatalismo que, por ejemplo, eximiría a todos los hombres de su responsabilidad moral.

Con el fin de mostrar cómo, desde las antípodas de los temores de Jacobi o Tschirnhaus, en Spinoza se produce una conciliación entre libertad y necesidad, en el primer apartado de este artículo realizaremos una sucinta exposición acerca de la revaloración de los afectos por parte de Spinoza. En la misma línea, el segundo apartado ahondará en la concepción del hombre como ser afectivo presentada en la Ética. A continuación, se caracteriza al hombre como ser determinado por otros modos y las afecciones que estos producen en él. Luego se presentarán los tres grados del conocimiento, que nos permitirá, al hablar del conocimiento intuitivo, introducir la noción de 'libertad necesaria' de Spinoza². Finalmente, se concluye que en la obra clave del autor holandés no solo está presente una comprensión de la libertad lo suficientemente amplia, sino que a partir de esta se plantea una determinada propuesta normativa.

\section{A favor de los afectos}

Lejos de la tradicional visión del hombre como el ente caracterizado por la posesión de un libre albedrío - de ser un imperium in imperio-, Spinoza afirma que el hombre no es una excepción en lo que al orden de la

\footnotetext{
${ }^{1}$ Como veremos, esta será propiamente la definición de libertad propuesta por Descartes, mas no por Spinoza. En una carta posterior el mismo autor de la Ética intentará sacar a Tschirnhaus de su error (Chaui, 2011, pp. 269-270).

2 También denominada 'verdadera libertad' (Kisner, 2011; Peña, 2018; Yu, 2020).
} 
naturaleza respecta (E, 3Pref.)3. Por lo contrario, a lo largo de la tradición numerosos autores han pretendido ver una realidad segregada entre el hombre y la naturaleza. Así, mientras que la última se erige como el reino de las pasiones, del vicio y de la necesidad, la primera contiene en sí la posibilidad de la libertad, pues hay una voluntad entendida como causa capaz de generar múltiples efectos (Chaui, 2011, p. 122). Por esto, la tradición teológico-metafísica con la que rompe Spinoza, desde Platón hasta Montaigne (Fernández, 1999, pp. 74-77), ha promovido una suerte de desdén, si no desprecio, por los afectos. Como consecuencia, no ha sido escasa la filosofía moralista entendida como ars moriendi en detrimento de la ars vivendi; en aras del olvido de los afectos, estos filósofos han edificado un "hombre del resentimiento, para quien toda felicidad es una ofensa y que hace de la miseria o la impotencia su única pasión" (Deleuze, 2009, p. 36)

En el mismo prefacio de la tercera parte de la Ética nuestro filósofo presentará a los llamados "hombres eminentes". A diferencia de la tradición teológico-metafísica, señalada en el anterior párrafo, estos hombres no vilipendiaron las pasiones humanas. No se trata de que los "hombres eminentes" ridiculicen o desprecien los afectos, sino que, de hecho, consideraron el problema con toda su gravedad. Su inconveniente reside en el otro lado, es decir, en su excesiva confianza en el poder de la razón. Pretendieron estos "hombres eminentes" hacer frente a las pasiones a través de un "imperium absolutum in nostras passiones" (E, 5 Pref.), es decir, a través de su dominación. Descartes y los estoicos serán

\footnotetext{
${ }^{3}$ Con el objetivo de respetar la citación consensuada de la Ética de Spinoza, en este artículo se combina la citación APA con aquella. Así, las referencias a la Ética podrán ser comprobadas en cualquier edición. En nuestro caso hemos seguido Spinoza, 2014. ${ }^{4}$ Como nos indica Deleuze, por esta misma causa Spinoza se encuentra en la estirpe crítica con las pasiones basadas en la tristeza, que va desde Epicuro hasta Nietzsche (2009, p. 68).
} 
paradigmáticamente los autores de esta índole. Al francés le reprochará Spinoza especialmente la relación jerárquica establecida entre cuerpo y alma: para Descartes el alma posee una libre voluntad que puede ejercer en el cuerpo a través de la glándula pineal y los celebérrimos esprits animaux. Muy por el contrario, para Spinoza "no hay en el alma [mens] ninguna voluntad absoluta o libre...” (E, 2P48).

Además, por supuesto, que la obra de Spinoza se caracterizará también por la ruptura de la primacía jerárquica del alma -en Spinoza más bien mente- sobre el cuerpo. La potencia de ambos se encuentra indisolublemente amalgamada y, por ende, carece de sentido contemplar la posibilidad de que, por ejemplo, la mente se encuentre activa mientras el cuerpo esté pasivo (E, 3P2, esc.). A los estoicos, Spinoza les achacará -a pesar de que podemos decir que Spinoza respira cierto aire estoico- la ingenuidad de considerar que nuestros afectos dependen de nosotros mismos, "de nuestra voluntad" y de que, por lo tanto, podamos domeñarlos a través de ella (E, 5 Pref.). En definitiva, Spinoza se rebelará contra la desconsideración de los afectos, sea cual fuere la vertiente de esta, para, a través de una concepción del hombre como ser preponderantemente afectivo, lograr una filosofía como ars vivendi, una filosofía de la alegría que nos conduzca al summun bonum y a la libertad (Fernández, 1988, pp. 75-81)5.

\section{El hombre como ser afectivo}

¿Qué es el hombre para Spinoza? Nos dice el autor de la Ética que el hombre es un modo finito más de entre los múltiples que componen el

\footnotetext{
${ }^{5}$ Estos motivos pudieran ya valernos para comprender el profundo rechazo provocado por la obra de Spinoza. No obstante, a esta apología de la alegría también habrá que sumarle la heterodoxa concepción de Dios, el rechazo del finalismo o la supresión de la libre voluntad (Chaui, 2011, p. 195).
} 
espesor ontológico del Deus sive natura; esto es, una afección de la substancia (Deus sive natura) que se encuentra entre la infinita selva de los demás modos que, asimismo, afectan a este (E, 1Def.). Así, el hombre es, en simultáneo, mente, en cuanto expresión del atributo pensamiento, y cuerpo, en cuanto expresión del atributo extensión y son, como sabemos, estos los dos atributos, de entre los infinitos existentes, que nuestro entendimiento percibe de la substancia. Como nos dice Margot, "si la sustancia se expresa en sus atributos, estos se expresan en sus modos" (2011, p. 34). Como hemos dicho, no existe una independencia entre cuerpo y mente, a diferencia de lo que autores previos al holandés habían pretendido ver. La mente y el cuerpo se encuentran en una interdependencia tal que, de hecho, la mente no es otra cosa que la idea del cuerpo, esto es, de las afecciones del cuerpo.

Vemos hasta aquí, pues, que somos un "cuerpo intercorporal” en relación constante, en coexistencia, con otros cuerpos externos, y es esta interrelación la que se manifiesta en la mente a través de las afecciones. De esto podemos extraer varias consecuencias: en primer lugar que, en virtud de los afectos, "mente y cuerpo son una sola y misma cosa" (E, $3 \mathrm{P} 2$, esc.). También que la "esencia de la mente consiste en afirmar la existencia de nuestro cuerpo" (E, 3 P10, dem.). Es decir, si tenemos conciencia de la existencia de nuestro cuerpo, es precisamente porque las afecciones que en él se producen, debido a sus relaciones con modos externos, afectan a la mente. De este modo, podemos derivar otro corolario de gran relevancia: aquello que aumenta o disminuye la potencia de obrar de nuestra mente aumenta o disminuye respectivamente la potencia de obrar de nuestro cuerpo (E, 3 P11).

Pero, más concretamente, ¿qué son los afectos?, o ¿a qué nos referimos cuando hablamos de la potencia de obrar? Los afectos los define nuestro 
filósofo como "las afecciones del cuerpo, por las cuales aumenta o disminuye, es favorecida o perjudicada, la potencia de obrar de ese mismo cuerpo, y entiendo, al mismo tiempo, las ideas de esas afecciones" ( $\mathrm{E}$, 3 Def.). Se extrae de aquí, pues, que mientras que la afección (affectio) se produce ante el inevitable contacto del hombre con lo externo, el afecto (affectus) es la idea misma de la variación producida por la afección. Se quiere decir, en su relación con los múltiples modos externos, que el hombre es afectado ora positiva ora negativamente. Positivamente en la medida en que resulta beneficiado, y por eso aquello que lo beneficia será lo bueno o útil, y negativamente en la medida en que resulta perjudicado, siendo la causa de que sea perjudicado lo malo. Spinoza llamará alegría al afecto que, derivado de una causa que entenderá como lo útil, hace pasar a la mente a una mayor perfección o potencia; la tristeza será lo contrario (E, 3 P11, esc.). Pero todavía existe, con todo, un afecto más primordial en el hombre: el deseo. El deseo (cupiditas) es ni más ni menos que la conciencia del hombre de su esfuerzo por perseverar en su ser, esto es, la conciencia del conatus (potentia sive conatus) que subyace a todas las cosas, su esencia siempre actual (E, 3 P6; $3 \mathrm{P} 7 ; 3 \mathrm{P} 9)$.

Los modos no son, en consecuencia, inertes. Todas las cosas tienden, se esfuerzan a mantenerse en su ser o, lo que es lo mismo, tienden a no ser destruidas necesariamente por otras cosas externas (E, $3 \mathrm{P} 4$ ). El hombre no es una excepción. El hombre, sometido a las mismas leyes de la naturaleza que los otros modos, quiere "durar por durar" (Álvarez, 1999, p. 141). Siempre en constante movimiento, el hombre está inmerso en una relación activa a varias partes: afectamos a lo demás, estos nos afectan a nosotros y nos autoafectamos. En cualquier caso, "el deseo es la esencia misma del hombre" (E, 3P9, esc.). Y, por supuesto, el deseo mismo es un afecto, el primero del que se derivan todos los demás; inmediatamente a la alegría y 
la tristeza. De todo lo dicho hasta ahora se sigue que lo alegre, lo bueno ${ }^{6} \mathrm{o}$ útil aumentarán nuestra potencia - tanto del cuerpo como de la mente- o, lo que es lo mismo, nuestra perfección.

Sin necesidad de entrar más pormenorizadamente en detalles, es menester introducir en este punto las dos modalidades de los afectos: la acción y la pasión. En el caso de que podamos ser causa adecuada de nuestras afecciones, afirma el autor de la Ética, estas serán acciones. Por la misma regla, si solo podemos ser su causa inadecuada o parcial, estas serán pasiones7. La diferencia entre ambas se manifiesta, así, en el grado en que nosotros mismos podamos ser la causa de la afección del cuerpo. Las acciones, afecciones en las que nosotros somos la causa adecuada, nos hacen "autónomos" en la medida en que estamos determinados por causas internas, por nosotros mismos. Por su parte, las pasiones nos hacen heterónomos, dependientes y desgraciados, pues estamos determinados por lo externo a semejanza de "las olas del mar agitadas por vientos contrarios” (E, 3P59, esc.). ¿Cuál es la solución al problema? Esta residirá en el fomento del conocimiento adecuado que permita transformar las pasiones en acciones.

\section{Los grados de conocimiento}

Parece que tenemos claro que debemos ser causa adecuada para poder aumentar nuestra potencia o perfección, lo cual que permitirá lograr, asimismo, el summun bonum, la suprema felicidad (beatitudo) y alegría; o, podemos adelantar, la libertad. Pero, ¿̇qué es, más específicamente, el

\footnotetext{
${ }^{6}$ Vemos, pues, cómo Spinoza deja de lado los absolutos bueno y malo en virtud de lo bueno y de lo malo. Esto responde a la crítica de Spinoza a la atribución de tales absolutos a Dios o la naturaleza. En otras palabras, siguiendo a Deleuze, la Ética como "tipología de los modos inmanentes de la existencia" reemplaza a la moral, "referida a la existencia de valores trascendentes" (2009, p. 34).

7 Lo cual no quiere decir que no pueda haber pasiones alegres, como por ejemplo el amor $(E, 3 P 13)$.
} 
conocimiento adecuado? Para responder a esta cuestión debemos remitirnos someramente a los tres géneros de conocimiento presentes en la Ética. Como es bien conocido, al primer género de conocimiento correspondiente a la "opinión" o "imaginación" pertenecen las "ideas inadecuadas o confusas" (E, 2P40, esc. II; 2P41). Estas ideas se dicen inadecuadas o parciales puesto que, a partir de ellas, tan solo conocemos vagamente las "modificaciones" de nuestro cuerpo, las afecciones a las que se encuentra sometido. Solo dan cuenta estas ideas confusamente de las afecciones del cuerpo, pero nada pueden decir acerca del origen de ellas. El problema se encuentra en que conocer, nos dice Spinoza, es conocer la causa - de otro modo estamos abocados a la heteronomía antes señalada- .

En consecuencia, este conocimiento de la imaginación, propio de los ignorantes, es la única "causa de la falsedad" (E, 2P41). En virtud de ella los hombres padecen, pues están sometidos a las pasiones, esto es, los hombres se encuentran obnoxius affectibus. Por mor de este conocimiento inadecuado a lo largo de la tradición los hombres, han caído en dos graves ilusiones o prejuicios:

1. En palabras de Deleuze, la "ilusión psicológica de la libertad" (2009, p. 73). Este prejuicio es una clara muestra de las consecuencias derivadas del conocimiento inadecuado: los hombres tienen la ilusión de que son libres, de que poseen una libre voluntad, debido simultáneamente al conocimiento de sus afecciones - resultantes de sus "choques" con los demás modos- y el desconocimiento de su causa (E, 2P35, esc.).

2. La "ilusión teológica de la finalidad", asimismo, ha propiciado "el alejamiento del verdadero conocimiento in vera rerum natura y nos ha empujado a buscar explicación de las cosas apoyándonos en las causas finales" (Alves Latournerie, 1999, p. 167). Unas causas finales 
generalmente consideradas como dispuestas por Dios - entendido como causa trascendente-. Sin más, como nos dice Jean-Paul Margot, en un universo necesario como el que nos bosqueja Spinoza la posibilidad de distinción "entre lo posible y lo real" es nula. No puede haber ni decisión ni finalidad puesto que todo lo real es ya siempre necesario (Margot, 2011, p. 31).

Vemos entonces la necesidad de dirigir la mirada a los dos géneros de conocimiento restantes. En el segundo género de conocimiento, el propio de la razón, ya nos encontramos con un conocimiento adecuado de las cosas (E, 2P40, esc. II), fundamentalmente por el conocimiento de las nociones comunes en las que tanto énfasis pondrá Deleuze (2009, pp. 113118). Todos los cuerpos, necesariamente, tienen algo en común y, asimismo, esto que tienen en común son necesariamente ideas adecuadas (E, 2P38; 2P39). En cualquier caso, la razón, en tanto "esencia de la mente cuando conoce clara y distintamente" (Chaui, 2011, p. 316), juega el papel de conocer las causas eficientes de las cosas con el fin de que nosotros mismos podamos ser causa adecuada de aquellas.

El objetivo es conocer el orden necesario de la naturaleza para, pudiendo ser nosotros mismos causa adecuada, transformar las pasiones que padecemos en acciones. De este modo, alcanzaremos finalmente el tercer género de conocimiento, la "ciencia intuitiva", que consiste en el conocimiento de la esencia eterna e infinita de Dios (E, 2P46), en el amor a Dios (amor Dei intellectualis). Así también, alcanzará el hombre (exemplar humanae naturae), mediante este conocimiento, la libertad 8 .

\footnotetext{
${ }^{8}$ Vemos con esto que, para alcanzar la libertad o felicidad, de lo que se trata es de transformar los afectos a los que necesariamente estamos abocados, no de ejercer un imperium absolutum in nostras passiones.
} 


\section{La sabiduría como garante de la libertad}

De lo dicho precedentemente se desprende el objetivo primero de la Ética: llevar al hombre de la servidumbre, o ignorancia, a la libertad, felicidad o alegría suprema por medio de la sabiduría. Para ello, acabamos de ver cómo la enfermedad y el diagnóstico están claros: los hombres padecemos en la medida en que somos afectados por pasiones ajenas, estamos por esto en una situación de servidumbre (servitude). A través del conocimiento de las causas eficientes, a saber, de origine et natura affectuum (de affectibus), debemos transformar las pasiones en acciones en las que nosotros mismos seamos autónomos (sui iuris). Sin embargo, un grave problema se nos presenta: el hombre es un modo finito más sometido a las relaciones con los demás y, en consecuencia, la "servidumbre es estructural e ineliminable" (Fernández, 1999, p. 101). Es decir, en ningún caso un hombre se puede erigir como un ser puro, infinito y libre como el Deus sive natura.

El hombre es finito y por eso no puede ser causa adecuada de todo lo que le sucede, de todos sus cambios (de potencia). Ahora bien, debemos distinguir esta servidumbre consustancial al humano (externe determinatur) de la servidumbre en la que el propio hombre es culpable por su ignorancia (interne determinatur). A través de la razón hemos visto que podemos reducir o tornar el conocimiento inadecuado en adecuado, las pasiones en acciones. Debemos pasar de ser alterius juris a sui juris.

Con todo, la proposición 7 de la parte IV parece que nos vuelve a complicar las cosas al demostrar que un afecto tan solo se puede eliminar a través de otro más fuerte $\left(\mathrm{E},{ }_{4} \mathrm{P} 7\right)$. Por esto mismo, la razón debe ser comprendida ella misma como un afecto. La virtud (E, $4 \mathrm{P}$, def.) será la potencia del hombre en la cual el deseo (como afecto primero) y la razón convergen. Esto es, la nuestra no es una razón fría o aséptica independiente de las 
afecciones tal como la han caracterizado una larga lista de moralistas, sino una "razón deseante", que es en sí misma el afecto más fuerte y fuente de la que emanan las acciones adecuadas (E, 4P58; 4P59; 4P61; 4P62). El hombre libre (exemplar humanae naturae) frente al sometido a la servidumbre de lo externo es libre "por sus afectos" y no "a pesar de ellos". Su libertad reside en su fortaleza (E, 3 P59, esc.), gracias a la cual actúa racionalmente a través del esfuerzo por "obrar bien y estar alegre" (E, 4P73, esc.) así como juntándose a los demás hombres en amistad (E, ${ }_{3} \mathrm{P}_{59}$, esc.) 9 ,

Con todo, la de Spinoza dista mucha de ser una mera oda al hombre libre. De lo que se trata, ya en la quinta parte de la Ética, en De libertate, es mostrar cómo lograr esa figura aparentemente tan utópica. Para ello se remarcará especialmente la potencia de nuestra mente sobre los afectos (E, 5 P2O, esc.). El fin será, como venimos diciendo, formarnos ideas claras y distintas, conocer la génesis de las pasiones para que dejen de ser tales (E, 5 P3). A través de este conocimiento no solo padeceremos menos, sino que transformaremos estas mismas pasiones en acciones por el propio conocimiento. El propio Spinoza pondrá el ejemplo de la ambición (E, $5 \mathrm{P} 4$, esc.). La ambición es una pasión alegre consistente en hacer exclusivamente aquello que complace a los hombres (E, 3 P29, esc.). Pues bien, en el hombre que vive bajo el dictamen de la razón esta pasión se torna, por su propio conocimiento, en la moralidad, en el deseo de hacer bien a los demás (E, 4P37, esc. I).

No obstante, parece claro que no se puede considerar precisamente liviana la tarea de alcanzar un "perfecto conocimiento de nuestros afectos". Por

\footnotetext{
${ }^{9}$ Esto se debe, cabe decir, a que nada es más útil para el hombre que los demás hombres que actúan "bajo la guía de la razón". Así, cuando esto sucede los hombres se prestan mutua ayuda uniéndose en amistad. A pesar de ello, el propio Spinoza nos advierte que raramente acaece que los "hombres vivan bajo la guía de la razón" ( $E$, 4P35, dem., cor. I, cor, II, esc.).
} 
este motivo, en el escolio de la proposición décima de la última parte de la Ética, nos propone nuestro filósofo una "norma recta de vida" (rectam vivendi rationem) que nos garantice unos "principios seguros" que nos sirvan de guía: "Por ejemplo, hemos establecido, entre los principios de la vida, que el odio debe ser vencido por el amor o la generosidad, y no compensado con odio" (E, 5 P10, esc.). De la misma forma, mientras aplicamos esta suerte de rutina vital, que ciertamente nos va aportando el conocimiento "de la virtud y sus causas", iremos dirigiendo "nuestras acciones según el imperio de la razón” (E, 5 P10, esc).

El esfuerzo del hombre que se quiera liberar de las ataduras de la servidumbre debe pasar por el conocimiento que aporta la "ciencia intuitiva", desembocando finalmente en el "mayor contento [acquiescentia] del alma" (E, 5 P27). La felicidad - ligada a la libertad-se manifiesta aquí como un punto clave, culminante, de este proceso de transformación de los afectos ${ }^{10}$. Como nos dice Eugenio Fernández: "Ella es la virtud misma y no un premio" (1999, p. 111). Así, mientras que en las éticas moralistas tradicionales la felicidad era el premio post mortem de una vida dedicada a la represión de los afectos ${ }^{11}$, en Spinoza la felicidad es el estado en el que se encuentra el sabio, ya libre, que ha sabido manejar sus afectos. Por ende, poco sentido tienen las quejas que Blijenbergh manifestaba en su correspondencia a Spinoza, especialmente la respectiva a la recompensa divina por una vida dedicada a la servidumbre (Chaui, 2011, pp. 259-260). Empero, es importante recordar una vez más que ni siquiera en el sabio libre desaparecen por completo las pasiones. Jamás un

\footnotetext{
${ }^{10}$ En el que conviene remarcar la importancia del cuerpo como se manifiesta en la demostración de la proposición 39 del De libertate.

11 Una de las más celebérrimas proposiciones de la Ética se encargará de demostrar que "un hombre libre en nada piensa menos que en la muerte" (E, 4P62). Incluso, se nos dirá que cuanto más conocimiento tengamos conforme al segundo y el tercero de los géneros de conocimiento tanto menos temeremos a la muerte ( $E, 5 P 38)$.
} 
modo finito podrá alcanzar la libertad absoluta, monopolizada por el Deus sive natura. Las pasiones son consustanciales a nosotros y "por eso siguen con nosotros, incomodando y alentando, dando quebraderos de cabeza y alegrías. Su destino no es diluirse en el silencio y descanso eternos. Ni el nuestro ser espíritus puros, plácidos ángeles apáticos” (Fernández, 1999, p. 112).

Es evidente, por tanto, que Spinoza no entiende la libertad del mismo modo que Descartes. La libertad no consiste en una ausencia de determinación en la medida en que tal ausencia es imposible. Siempre nos topamos determinados. Ahora bien, no es menos cierto que existe una importante diferencia, en la que Spinoza pone el acento, entre la determinación externa y la interna. Chaui caracteriza esta libertad como "una conquista contra la fortuna en el campo de la fortuna" (Chaui, 2011, p. 202). De lo que se trata es de ser libre siendo nosotros nuestra propia determinación. Una acción es libre en el momento en que esté "internamente determinada por la necesidad de nuestra naturaleza" (Chaui, 2011, p. 283).

Esta es la concepción de libertad manejada por Spinoza, la llamada "libertad necesaria" (Chaui, 2011, p. 273) que tanto escandalizó a Jacobi. El hombre libre, al conocer el origen de sus afecciones, únicamente se encuentra autodeterminado hasta el punto de que "no permitirá que los pensamientos y deseos de otros dicten lo que él piensa y quiere" (Bennett, 1990, p. 331). Pero ¿cómo lograr en su zenit esta "libertad necesaria"? Como hemos mencionado, siguiendo la guía de la razón hasta alcanzar el mayor conocimiento posible, el conocimiento del entramado inmanente y necesario del que el hombre forma parte (sub specie aeternitatis), esto es, la esencia misma del Deus sive matura. Se conoce así la determinación a la que estamos sometidos buscando una "totalidad de control voluntario" 
(Bennett, 1990, p. 334). En este momento de amor a Dios, de amor intelectual, de aceptación de la necesidad natural, el hombre habrá alcanzado el conocimiento intuitivo pasando del estado de servidumbre (obnoxius fortunae potestate) al de libertad. Es este, además, el punto en el que se logrará, por fin, una armoniosa relación entre lo que es y lo que debe ser, así como entre el concepto y el afecto (Deleuze, 2009, p. 158). No serán pocas las críticas a la noción de "libertad necesaria" esbozada ${ }^{12}$. Ciertamente, cabe reconocer que algunas de las consecuencias que Spinoza extrae de la mentada noción resultan seriamente cuestionables. Serán de especial interés las críticas recibidas por su ya nombrado coetáneo Tschirnhaus. Ciertamente, sabemos que estas críticas fueron respondidas una por una por el autor de la Ética. En general, una de las vertientes en la que se muestra la acusación fatalista cuestiona, en virtud de la doctrina spinozista, la ausencia de responsabilidad moral: "Além disto, se assim fosse, toda maldade estaría desculpada”, nos dice Tschirnhaus (Chaui, 2011, p. 268). Pero nada más lejos de la realidad; de la propuesta de Spinoza, no se sigue que los hombres nos topemos en una suerte de quietud resignada. Aun a pesar de su concepción ontológica, Spinoza consigue conjugar lo que es -nuestra determinación en tanto modos finitos- con lo que debe ser -el hombre libre guiado/determinado por la razón que, entre otras cosas, abandera afectos como la firmeza, la generosidad, la pietas o la moralidad-. Todavía más, lejos de promover un olvido de nuestros deberes para con los demás, la ética de Spinoza - un ilustrado avant la lettre - remarcará la ayuda al prójimo precisamente bajo la guía de la razón.

\footnotetext{
12 Para una crítica más contemporánea a esta concepción de la libertad véase la propuesta por Isaiah Berlin en su célebre Two Concepts of Liberty (1958), sintetizado magistralmente por De Toffoli (2019).
} 


\section{Conclusiones}

Spinoza se opondrá a una dilatada tradición, paradigmáticamente representada por Descartes, que venía presentando a la naturaleza humana preponderantemente como una entidad racional que debía domeñar su dimensión pasional, sometida a los "instintos naturales". Esto es, la naturaleza es un lugar irracional e inmoral que hay que contrarrestar. Venían predicando los moralistas que debemos construir una frontera entre nosotros y nuestros afectos. Spinoza será quien muestre la imposibilidad de tal empresa al no ser el hombre otra cosa que un ser afectivo. Desechando estas visiones ficticias del hombre, el autor de la Ética consigue cimentar una concepción del ser más acorde a la naturaleza humana. Para el caso, una concepción donde la libre voluntad, tal y como se venía entendiendo, se muestra como una ficción.

No obstante, lejos de abandonarnos en una suerte de mundo amoral Spinoza conseguirá lograr una perfecta armonía de este ser, depurado de vetustas fantasías, con el deber ser. Un deber ser que ya no se presenta, consecuentemente, como una contradicción con el propio ser. Ya no se trata de renegar de nuestra propia naturaleza -ni de la naturaleza en general-, sino de aceptarla gracias al conocimiento que nos proporciona la razón, el conocimiento más excelso, el intuitivo. Así, ya no solo no se trata de que Spinoza nos conduzca a un "fatalismo del todo indeseable", como se temía Jacobi, sino que las consecuencias de su filosofía resultan del todo positivas.

A modo de ejemplo, Spinoza nos presenta cuatro beneficios (E, 4P49, esc.). Primeramente, esta doctrina de la "libertad necesaria" tal y como la hemos visto nos confiere un "completo sosiego" ante el conocimiento de nuestra condición en el Deus sive natura; así como la comprensión de lo absurdo de esperar una recompensa divina por nuestras "buenas acciones". En 
segundo lugar, el sosiego proporcionado por esta doctrina se extiende hasta el punto de que debemos, por nuestra propia felicidad, contemplar con "ánimo equilibrado" los sucesos de la fortuna que no caen bajo nuestra potestad. De la misma manera, también es esta doctrina útil para la vida social, esto es, a la hora de relacionarnos con los demás. Puesto que conocemos su situación de modos finitos, determinados, como individuos que nos hallamos bajo la guía de la razón, nos abstendremos en la medida de nuestras posibilidades de, por ejemplo, odiar, envidiar, burlar o despreciar a los demás. Por último, esta doctrina también será útil en el plano político. Por ejemplo, para que los gobernantes comprendan que no se trata de dirigir a los ciudadanos "para que sean siervos, sino para que hagan libremente lo mejor" (E, 4P49, esc.).

Vemos que no son pocos los beneficios que la noción de "libertad necesaria", manejada por Spinoza, nos pueden proporcionar, tanto a nivel individual como colectivo (Taylor, 2020). La cuestión de lograrlo, nunca de manera plena, es asunto de cada uno, ya que como sentencia nuestro filósofo "todo lo excelso es tan difícil como raro" (E, 5 P42, esc.).

En el presente trabajo se ha visto cómo, lejos de ser conceptos irreconciliables, libertad y necesidad se encuentran en el marco del sistema spinoziano indisolublemente amalgamados. De hecho, este corolario puede llevarse a tal punto radical que una correcta comprensión de la antropología, ontología y ética de Spinoza no deja lugar, como hemos intentado mostrar, a ninguna otra interpretación.

Para finalizar, nada más queda por decir que tal armonioso edificio intelectual construido por este filósofo proporciona un soplo de aire fresco en el mundo actual. Un mundo donde, permítasenos decir, predomina en el imaginario colectivo el desdén por la figura del sabio y que se encuentra atorado de numerosas consideraciones acerca de los afectos huérfanas de 
valor intelectual. En un mundo así, la reflexión spinoziana sobre el ser humano, sus afectos y pasiones, la libertad... resulta extremadamente necesaria.

\section{Referencias}

Álvarez, A. (1999). El místico y el rebelde: dos modos de ser racionalista. En J. M.BlancoEchauri (Ed.), Espinosa: Ética e Política. Encontro Hispano-Portugués de Filosofía, 5-7 de abril de 1997 (pp. 137-164). Universidad de Santiago de Compostela.

Alves Latournerie, E. (1999). Los modos, operarios de la fábrica spinozista. En J. M. Blanco-Echauri (Ed.), Espinosa: Ética e Política. Encontro Hispano-Portugués de Filosofía, 5-7 de abril de 1997 (pp. 165-174). Universidade de Santiago de Compostela.

Barreiro, X. L. (1999). Ilustración y fideísmo. La polémica Jacobi-Lessing y sus repercusiones. En J. M. Blanco-Echauri (Ed.), Espinosa: Ética e Política. Encontro Hispano-Portugués de Filosofía, 5-7 de abril de 1997 (pp. 175-189). Universidad de Santiago de Compostela.

Bennett, J. (1990). Un estudio de la Ética de Spinoza. Fondo de Cultura Económica.

Chaui, M. (2011). Desejo, paixāo e açāo na ética de Espinosa. Companhia das Letras.

Deleuze, G. (2009). Spinoza: filosofía práctica. Tusquets.

Fernández, E. (1999). Dominio de los afectos. En J. M. Blanco-Echauri (Ed.), Espinosa: Ética e Política. Encontro Hispano-Portugués de Filosofía, 5-7 de abril de 1997 (pp. 73-112). Universidad de Santiago de Compostela.

Kisner, M. J. (2011). Spinoza on Human Freedom: Reason, Autonomy and the Good Life. Cambridge University Press.

Margot, J. P. (2011). Libertad y necesidad en Spinoza. Praxis Filosófica, 32, 27-44. https://doi.org/10.25100/pfilosofica.voi32.3437

Spinoza, B. (2014). Ética demostrada según el orden geométrico. Alianza.

Taylor, D. (2020). Spinoza and the Politics of Freedom. Edinburgh University Press. Yu, A. (2020). Spinoza on Human Freedom [tesis de maestría]. University of Chicago. 